\title{
On a construction of self-dual gauge fields in seven dimensions
}

\author{
E K LOGINOV ${ }^{a}$ and $A N$ GRISHKOV ${ }^{b}$ \\ ${ }^{a}$ Department of Physics, Ivanovo State University \\ Ermaka St. 39, Ivanovo, 153025, Russia \\ E-mail: ek.loginov@mail.ru \\ ${ }^{b}$ IME, University of Sao Paulo \\ CER 005598-300, Sao Paulo, Brazil \\ E-mail: grishkov@ime.usp.br
}

Received April 25, 2007; Accepted in Revised Form June 27, 2007

\begin{abstract}
We consider gauge fields associated with a semisimple Malcev algebra. We construct a gauge-invariant Lagrangian and found a solution of modified Yang-Mills equations in seven dimensions.
\end{abstract}

\section{Introduction}

In Ref. [1], the 4d (anti-)self-dual Yang-Mills equations were generalized to the higherdimensional linear relations

$$
c_{m n p s} F^{p s}=\lambda F_{m n}
$$

where the numerical tensor $c_{m n p s}$ is completely antisymmetric and $\lambda=$ const is a non-zero eigenvalue. It is obvious that these equations lead to the full Yang-Mills equation, via the Bianchi identity. Several anti-self-dual solutions of (1.1) were found in [2-6]. Conversely, self-dual solutions of (1.1) are known. However, if we assume that the tensor $F_{m n}$ may take values in a Malcev algebra, then such solution may be found. Interestingly that this solution exactly coincides with the general solution of $7 d$ analogue of the $3 d$ Euler top equation [7]. The $7 d$ model is regarded as a model describing self-dual membran instantons [8].

The paper is organized as follows. Section 2 contains well-known facts about CayleyDickson algebras, Malcev algebras, and analytic Moufang loops which we use. In Sections 3 gauge fields associated with a semisimple Malcev algebra are investigated, and a gaugeinvariant Lagrangian of the Yang-Mills type is constructed. In Section 4 solutions of equations of motion in seven dimensions are found. 


\section{Moufang loops and Malcev algebras}

Recall that a loop is a binary system $S$ with an unity element, in which the equations $a x=b$ and $y a=b$ are uniquely solvable for all $a, b \in S$. Moufang loops are distinguished from the class of all loops by the identity

$$
(x y)(z x)=(x(y z)) x .
$$

This paper is concerned with connected analytic Moufang loops; that is, connected analytic manifolds equipped with the Moufang loop structure, in which the binary operations are analytic. Everywhere below for short we use the term "Moufang loop" in place of the term "connected analytic Moufang loop".

Moufang loops are closely associated with alternative algebras that are defined by the identities

$$
x^{2} y=x(x y), \quad y x^{2}=(y x) x .
$$

It is evident from the definition that any associative algebra is alternative. The most important example of a nonassociative alternative algebra is the real Cayley-Dickson algebra. Let us recall its construction (see [9]).

Let $A$ be a real linear space equipped with a nondenerate symmetric metric $g$ of signature $(8,0)$ or $(4,4)$. Choose the basis $\left\{1, e_{1}, \ldots, e_{7}\right\}$ in $A$ such that

$$
g=\operatorname{diag}(1,1,1,1, \pm 1, \pm 1, \pm 1, \pm 1),
$$

and define the multiplication

$$
e_{i} e_{j}=-g_{i j}+c_{i j}{ }^{k} e_{k},
$$

where the structure constants $c_{i j k}=c_{i j}{ }^{s} g_{s k}$ are completely antisymmetric and different from 0 only if

$$
c_{123}=c_{145}=c_{167}=c_{246}=c_{275}=c_{374}=c_{365}=1 .
$$

The multiplication (2.2) transform $A$ into a linear algebra that is called the Cayley-Dickson algebra.

In general, the commutator algebra $A^{(-)}$of the alternative algebra $A$ is not a Lie algebra. Instead of the Jacobi identity, $A^{(-)}$satisfies the identity

$$
J(x, y,[x, z])=[J(x, y, z), x],
$$

where $J(x, y, z)$ is so-called Jacobian of the elements $x, y, z$

$$
J(x, y, z)=[[x, y], z]+[[y, z], x]+[[z, x], y] .
$$

An anticommutative algebra whose multiplication satisfies the identity (2.3) is called a Malcev algebra. Everywhere below we use the term "Malcev algebra" in place of the term "finite-dimensional real Malcev algebra".

Malcev algebras and alternative algebras are closely associated [10-12]. Any nonLie simple Malcev algebra is isomorphic to the quotient algebra $A^{(-)} / \mathbb{R}$, where $A$ is a Cayley-Dickson algebra. Any semisimple Malcev algebra $M$ is decomposed in the direct 
sum $M=N(M) \oplus J(M)$ of Lie center $N(M)$ and ideal $J(M)$ generated by Jacobians. Besides, $N(M)$ is a semisimple Lie algebra and $J(M)$ is a direct sum of non-Lie simple Malcev algebras. In particular, it follows from here that any semisimple Malcev algebra is embedded in the commutator algebra of an appropriate alternative algebra.

Further, recall that a derivation of a Malcev algebra $M$ is a linear transformation $D$ of $M$ satisfying

$$
D[x, y]=[D x, y]+[x, D y]
$$

for all $x, y \in M$. We may write it in an equivalent form

$$
\left[D, T_{x}\right]=T_{D x}
$$

where $T: M \rightarrow \operatorname{End}(M)$ is the regular representation of $M$, such that $T_{x} y=[x, y]$.

We denote by $\operatorname{Lie}(M)$ the Lie algebra generated by all operators $T_{x}$. If $M$ is a semisimple Malcev algebra, then $\operatorname{Lie}(M)$ is a direct sum of the derivation subalgebra $\operatorname{Der}(M)=\operatorname{Der}(N) \oplus \operatorname{Der}(J)$ and vector subspace $T(M) \simeq J(M)$. In addition, the subalgebra $\operatorname{Der}(N) \simeq N(M)$ and the subalgebra $\operatorname{Der}(J)$ is linearly generated by the operators

$$
D(x, y)=T_{[x, y]}+\left[T_{x}, T_{y}\right]
$$

where $x, y \in J(M)$. With the regular representation $T$ we may connect the bilinear form $(x, y)=\operatorname{tr}\left(T_{x} T_{y}\right)$. It is clear that the form $(x, y)$ is symmetric $(x, y)=(y, x)$. In addition, it follows from (2.3) that

$$
([x, y], z)=(x,[y, z])
$$

for any $x, y, z \in M$. The form $(x, y)$ is called a Killing form.

The regular representation of Malcev algebras can be extended. Let $M$ be a Malcev algebra, $V$ be a real vector space, and $\tau: M \rightarrow \operatorname{End}(V), x \rightarrow \tau_{x}$ a linear mapping. Then $\tau$ is called a representation of $M$ if the algebra defined on the space $M \oplus V$ by

$$
[x+v, y+w]=[x, y]+\tau_{x} w-\tau_{y} v
$$

is a Malcev algebra. In this case $V$ is called a Malcev module. It is known [13-14] that every representation of a semisimple Malcev algebra is completely reducible. Any irreducible Malcev module is either Lie or the regular bimodule for a nonassociative simple Malcev algebra or $s l(2)$-module of dimension 2 such that $\tau_{x}=\bar{x}$, where $\bar{x}$ is the adjoint matrix to $x \in \operatorname{sl}(2)$.

Finally, we note that there exists a correspondence between Moufang loops and Malcev algebras, which generalizes the classical Lie correspondence between Lie groups and Lie algebras [15-17]. Namely, there exists an unique, to within isomorphism, simply connected Moufang loop $S$ with a given tangent Malcev algebra, and any Moufang loop with the same tangent algebra is isomorphic to the quotient algebra $S / N$ where $N$ is a discrete central normal subgroup of $S$. Any simply connected semisimple Moufang loop is decomposed in the direct product of a semisimple Lie group and a simple nonassociative Moufang loops each of which is analytically isomorphic to one of the spaces $\mathbb{S}^{7}, \mathbb{S}^{3} \times \mathbb{R}^{4}$, or $\mathbb{S}^{7} \times \mathbb{R}^{7}$. Actually, any simply connected simple nonassociative Moufang loop is isomorphic to the loop of elements of norm 1 in the Cayley-Dickson algebra over $\mathbb{R}$ or $\mathbb{C}$. 
Using the previously mentioned correspondence between Moufang loops and Malcev algebras, we can define a notion of the representation of Moufang loop. Let $S$ be a Moufang loop and $M$ be its tangent Malcev algebra. The representation $M \rightarrow \operatorname{End}(V)$ of $M$ induces the mapping $\widetilde{S} \rightarrow \operatorname{Aut}(V)$ of the simply connected Moufang loop $\widetilde{S}$, locally isomorphic to $S$, into the simply connected Lie group $\widetilde{G} \subseteq A u t(V)$. This mapping can extend into the group $G \simeq \widetilde{G} / \widetilde{G}_{0}$, where the subgroup $\widetilde{G}_{0}$ lies in the discrete center of $\widetilde{G}$. Therefore if $S \simeq \widetilde{S} / \widetilde{S}_{0}$ and $\widetilde{S}_{0}$ is isomorphic to a subgroup of $\widetilde{G}_{0}$, then there exists the mapping $S \rightarrow G$ induced by the representation of $M$. We shall call this mapping by a representation of $S$. In particular, if $S$ is a nonassociative compact simple Moufang loop, then $\widetilde{G} \simeq \operatorname{Spin}(7)$ and $G \simeq S O(7)$.

\section{$3 \quad$ Nonassociative gauge fields}

Suppose $S$ is a semisimple Moufang loop, $M$ is its tangent Malcev algebra, and $A_{m}(x)$ is a vector field taking values in $M$ and defined in an Euclidean or pseudo-Euclidean space. Further, let

$$
S \rightarrow G \subseteq A u t(V)
$$

be a representation of $S$, and $H$ be a subgroup of $G$ locally isomorphic to the group $\operatorname{Int}(S)$ of inner automorphisms of $S$. Define the field $\psi(x)$ taking values in $V$ and its covariant derivative

$$
\hat{D}_{m} \psi=\left(\partial_{m}+\hat{A}_{m}\right) \psi
$$

where $\hat{A}_{m} \in \operatorname{End}(V)$ is an image of $A_{m}$ corresponding to the representation (3.1). As usually, we require that $\hat{D}_{m} \psi$ has transformation properties the same as the field $\psi$, i.e.

$$
\begin{aligned}
\psi & \rightarrow \psi^{\prime}=\hat{u} \psi, \\
\hat{D}_{m} \psi & \rightarrow \hat{D}_{m}^{\prime} \psi^{\prime}=\hat{u} \hat{D}_{m} \psi,
\end{aligned}
$$

where $\hat{u}(x)$ is function taking values in the subgroup $H$.

Consider an infinitesimal gauge transformation $\hat{u}=1+\hat{\varepsilon}$. It is obvious that $\hat{\varepsilon} \in$ $\operatorname{Der}(M)$. If the field $A_{m}$ takes values in the Lie center $N(M)$, then the condition (3.2) defines the usual transformation rule of gauge fields

$$
A_{m} \rightarrow A_{m}+\left[\varepsilon, A_{m}\right]-\partial_{m} \varepsilon
$$

where $\varepsilon$ is an isomorphic prototype of $\hat{\varepsilon}$ in $N(M)$. If $A_{m}$ is an element of the ideal $J(M)$ generated by Jacobians, then the situation is different. In this case the operator $\hat{\varepsilon}$ has not a prototype in $M$. On the other hand, the representation of $J(M)$ induced by the representation of $S$ is regular. Therefore we may use the identity (2.5) and get the following transformation rule

$$
\left.\begin{array}{l}
\partial_{m} \rightarrow \partial_{m}-\partial_{m} \hat{\varepsilon}, \\
A_{m} \rightarrow A_{m}+\hat{\varepsilon} A_{m}
\end{array}\right\} .
$$


Obviously, the transformation (3.4) of $A_{m}$ induces a transformation of $\hat{A}_{m}$ according to the rule

$$
\hat{A}_{m} \rightarrow \hat{u} \hat{A}_{m} \hat{u}^{-1} .
$$

Now we go on to a construction of the gauge-invariant Lagrangian. To this end define tensor fields in $M$ such that their images in $\operatorname{End}(V)$ are transformed by the adjoined representation of $G$. This request is satisfied if we suppose

$$
\begin{aligned}
F_{m n} & =\partial_{m} A_{n}-\partial_{n} A_{m}+\left[A_{m}, A_{n}\right], \\
J_{m} & =\frac{1}{12} c_{m n p s} J\left(A^{n}, A^{p}, A^{s}\right),
\end{aligned}
$$

where $c_{\text {mnps }}$ is a completely antisymmetric numerical tensor. Indeed, it can easily be checked that the infinitesimal transformations (3.3) and (3.4) induce the transformations

$$
\begin{array}{rlrl}
F_{m n} & \rightarrow F_{m n}+\hat{\varepsilon} F_{m n}, & & J_{m} \rightarrow J_{m}+\hat{\varepsilon} J_{m}, \\
\hat{F}_{m n} \rightarrow \hat{u} \hat{F}_{m n} \hat{u}^{-1}, & \hat{J}_{m} \rightarrow \hat{u} \hat{J}_{m} \hat{u}^{-1},
\end{array}
$$

where $\hat{F}_{m n}$ and $\hat{J}_{m}$ are images of $F_{m n}$ and $J_{m}$, which are defined by the representation (3.1). Note that the tensor $F_{m n}$ extend a notion of the Yang-Mills field strength. Conversely, the field $J_{m}$ has not a prototype in the Yang-Mills theory. The field $J_{m}$ is not zero only if $M$ is a non-Lie Malcev algebra.

We define the Lagrangian

$$
L=\frac{1}{8 g^{2}} \operatorname{tr}\left(\hat{F}_{m n} \hat{F}^{m n}+\hat{J}_{s} \hat{A}^{s}\right) .
$$

If $M$ is a Lie algebra, then the Lagrangian (3.7) coincides with the Lagrangian of YangMills and hence it is gauge-invariant. On the other hand, every representation of a semisimple Malcev algebra is completely reducible. Therefore it is enough to prove $H$-invariance of the Lagrangian (3.7) only if $M$ is a non-Lie simple Malcev algebra. But in this case the gauge-invariance of the Lagrangian (3.7) follows from (3.5) and (3.6).

\section{Solutions of the equations of motion}

Let $M$ be a non-Lie simple Malcev algebra. Then $M \simeq A^{(-)} / \mathbb{R}$, where $A$ is a CayleyDickson algebra, and hence the algebra $M$ admits the basis $e_{1}, \ldots, e_{7}$ with the multiplication table

$$
\left[e_{i}, e_{j}\right]=2 c_{i j}{ }^{k} e_{k} .
$$

In this basis the operators $T_{e_{i}}$ are represented in the form

$$
T_{e_{i}}=c_{i}{ }^{j k} e_{j k},
$$

where $e_{j k}$ are generators of the Lie algebra $\operatorname{Lie}(M) \simeq s o(7)$ or $s o(3,4)$ with the matrix elements

$$
\left(e_{j k}\right)_{b}^{a}=g_{j b} \delta_{k}^{a}-g_{k b} \delta_{j}^{a} .
$$


Using the representation (4.1) and the identity (4.2), we can find the Killing form

$$
\left(e_{i}, e_{j}\right)=-24 g_{i j} .
$$

Substituting (4.3) in the Lagrangian (3.7), we easily get the equations of motion

$$
\partial^{m} F_{m n}+\left[A^{m}, F_{m n}\right]=J_{n} .
$$

Using the definition (2.4), we prove that every solution of the equations

$$
F_{m n}=\frac{1}{4} c_{m n p s} F^{p s}
$$

is a solution of the equations of motion (4.4).

Further, let $H$ be a Lie group locally isomorphic to the group $\operatorname{Int}(M)$ of inner automorphisms of $M$. Then $H \simeq G_{2}$ (or $H \simeq G_{2}^{\prime}$ in noncompact case). Define by

$$
J\left(e_{i}, e_{j}, e_{k}\right)=12 c_{i j k}^{l} e_{l}
$$

the completely antisymmetric $H$-invariant tensor $c_{i j k l}$. If we write out its nonzero components

$$
c_{4567}=c_{2367}=c_{2345}=c_{1357}=c_{1364}=c_{1265}=c_{1274}=1,
$$

then it is easy to prove that the tensors $c_{i j k}$ and $c_{i j k l}$ satisfy the following identities

$$
\begin{aligned}
c_{m n i} c_{p s}{ }^{i} & =g_{m p} g_{n s}-g_{m s} g_{n p}+c_{m n p s}, \\
c_{m n i} c_{p s j}{ }^{i} & =c_{p s[m} g_{n] j}+c_{s j[m} g_{n] p}+c_{j p[m} g_{n] s}, \\
c_{m n i j} c_{p}^{i j} & =4 c_{m n p}, \\
c_{m n i j} c_{p s}{ }^{i j} & =4 g_{m p} g_{n s}-4 g_{m s} g_{n p}+2 c_{m n p s} .
\end{aligned}
$$

Note that if the gauge fields are defined in an Euclidean space and $M$ is a compact algebra, then any solution of (4.5) correspond to a minimum of the functional of action. Indeed, using the identities (2.7) and (4.9), we can shown that

$$
L=\frac{1}{8 g^{2}} \operatorname{tr}\left[\frac{2}{3}\left(\hat{F}_{m n}-\frac{1}{4} c_{m n p s} \hat{F}^{p s}\right)^{2}+\partial^{m} \hat{I}_{m}\right],
$$

where the prototype of $\hat{I}_{m}$ in $M$ has the form

$$
I_{m}=c_{m n p s}\left(A^{n} \partial^{p} A^{s}+\frac{2}{3}\left(A^{n} A^{p}\right) A^{s}\right) .
$$

Since the variation of field on the boundary of volume vanish in the deduction of equations of motion, we get the required statement.

Now we turn to a search of solutions of the equations (4.5). Suppose that the field $F_{m n}$ is defined in the space $M$ with the metric (2.1). We choose the ansatz

$$
A_{m}(x)=\frac{c_{m i j} x^{j}}{\lambda^{2}+x^{2}} e^{i},
$$


where $x^{2}=x_{k} x^{k}$. Using the identities (4.6)-(4.7), we get the following expression of field strength

$$
F_{m n}(x)=\frac{2 c_{m n i}\left(\delta_{j}^{i} \lambda^{2}+2 x^{i} x_{j}\right)}{\left(\lambda^{2}+x^{2}\right)^{2}} e^{j} .
$$

By the identity (4.8), it follows that the tensor (4.11) is self-dual

The obtained solution may be generalized. We choose the ansatz

$$
A_{m}(x)=6 c_{m i j} x^{i} B^{j}(u),
$$

where depending on $u=\lambda^{2}+x^{2}$ the vector $B^{j}$ takes values in $M$. If the vector $B_{i}$ satisfies the identity

$$
\frac{d B_{i}}{d u}=\frac{1}{2} c_{i j k}\left[B^{j}, B^{k}\right],
$$

then the field strength again is self-dual

$$
F_{m n}(x)=12 c_{m n j}\left(B^{j}+\frac{d B^{j}}{d u} x^{2}+\frac{d B_{i}}{d u} x^{i} x^{j}\right) .
$$

A particular solution of the equation (4.13) can find if to compare the right parts of (4.10) and (4.12). General solution of (4.13) was found in the work [7].

Acknowledgments. E.K. Loginov is grateful to the foundations RFBR 06-02-16140 and FAPESP 06/61138-6 for support. A.N. Grishkov thanks FAPESP 05/60337-2 and 05/00666-2 for partial support.

\section{References}

[1] Corrigan E, Devchand C, Fairlie D B and J. Nuyts, First-order equations for gauge fields in spaces of dimension greater than four, Nucl. Phys. B 214 (1983) $452-465$.

[2] FAirlie D B and J. NuYts, Spherically symmetric solutions of gauge theories in eight dimensions, J. Phys. A 17 (1984) 2867-2872.

[3] Fubini S and Nicolai H, The octonionic instantons, Phys. Lett. B 155 (1985) 369-372.

[4] Ivanova T A and Popov A D, Self-dual Yang-Mills fields in $d=7,8$, octonions and Ward equations, Lett. Math. Phys. 24 (1992) 85-92.

[5] Loginov E K, Self-dual Yang-Mills fields in pseudo-Euclidean spaces, J. Phys. A 37 (2004) 6599-6604.

[6] Loginov E K, Multi-instantons in seven dimensions, J. Math. Phys. 46 (2005) 063506. 
[7] Ueno T, General solution of 7-D octonionic top equation, Phys. Lett. A 245 (1998) $373-381$.

[8] Floratos E G, Leontaris G K, Polychronakos A P and Tzani R, On the instanton solutions of the self-dual membrane in various dimensions, Phys. Lett. B421 (1998) 125-130.

[9] Schafer R D, An introduction to non-assocoative algebras, Acad. Press, New York, 1966.

[10] Sagle A A, Malcev algebras, Trans. Am. Math. Soc. 101 (1961) 426-458.

[11] Kuzmin E N, Malcev algebras and their representations (Russian), Algebra and Logic 7 (1968) 48-69.

[12] Elduque A, On semi-simple Malcev algebras, Proc. Amer. Math. Soc. 107 (1989) $73-82$.

[13] Carlson R, On the exceptional central simple non-Lie Malcev algebras, J. Reine Anew. Math. 281 (1976) 199-210.

[14] Elduque A, On Malcev modules, Comm. Algebra 18 (1990) 1551-1561.

[15] Malcev A I, Analytic loops (Russian), Math. Sb. 36 (1955) 569-576.

[16] Kuzmin E N, On a connection between Malcev algebras and analytic Moufang loops (Russian), Algebra and Logic 10 (1971) 3-22.

[17] Kerdman F S, On analytic Moufang loops in the large (Russian), Algebra and Logic 18 (1979) 523-555. 